\title{
Eliciting ELT Students' Understanding of Plagiarism in Academic Writing
}

\author{
Irina Rets ${ }^{a} *$ (D), Ali Ilya $b+(D)$ \\ ${ }^{a}$ The Open University, Milton Keynes, MK7 6AA, United Kingdom \\ ${ }^{b}$ Sakarya University, Sakarya, 54050, Turkey
}

Received 15 April 2018 | Received in revised form 05 July 2018 | Accepted 24 September 2018

\begin{abstract}
APA Citation:
Rets, I. \& Ilya, A. (2018). Eliciting ELT students' understanding of plagiarism in academic writing. Eurasian Journal of Applied Linguistics, 4(2), 193-211. doi: 10.32601/ejal.464115
\end{abstract}

\begin{abstract}
Given that the term 'plagiarism' is open to multiple interpretations, resulting in confusion among students and teachers alike, research that investigates the current state of empirical evidence and sheds light on students' ability to define and detect this notion has important pedagogical implications. This study examines undergraduate English Language Teaching (ELT) students' understanding of plagiarism in academic writing through qualitative data collection methods. After the focus group filled in the openended questionnaire, they were exposed to two sets of texts each containing an original, a plagiarized and non-plagiarized copy. The copy in the first set featured mainly word-for-word plagiarism while the copy in the second set was plagiarized in terms of illicit paraphrasing. The students were asked to identify whether there is any plagiarism in each copy and assess the texts regarding their acceptability in the format of an interview and think-aloud protocols. The results of the open-ended questionnaire and interviews were compared revealing that although all the students were able to define plagiarism correctly, most of them failed to identify it in the written text. The study also uncovered discrepancies in how the students view the aforementioned types of plagiarism.
\end{abstract}

(C) 2018 EJAL \& the Authors. Published by Eurasian Journal of Applied Linguistics (EJAL). This is an open-access article distributed under the terms and conditions of the Creative Commons Attribution license (CC BY-NC-ND) (http://creativecommons.org/licenses/by-nc-nd/4.0/).

Keywords: Paraphrasing and unacknowledged copying; plagiarism; scientific writing; think-aloud protocols; undergraduate research experience.

\section{Introduction}

\subsection{Defining plagiarism}

The concept of plagiarism has been studied from different perspectives across a broad range of disciplines. Originally derived from the Latin term 'plagiare' meaning 'kidnapping', plagiarism is defined as the action of 'using words, ideas, or work products attributable to another identifiable person or source without attributing the work to the source from which it was obtained' (Fishman, 2009, p. 5). Although, there

\footnotetext{
* Corresponding author. Tel.: +44-190-865-4938

E-mail address: irina.rets@open.ac.uk

† ailya@sakarya.edu.tr
} 
seems to be a consensus that plagiarism is associated with the absence of credit to the original source, the availability of multiple definitions of this notion as well as ranging views on the degree of seriousness indicate that the understanding of plagiarism differs widely. The mere fact that the term 'plagiarism' is used alongside and often interchangeably with other terms such as 'academic dishonesty', 'cheating', 'academic misconduct', 'copying', 'compromise of academic integrity' and even 'intertextuality' which is defined as 'shaping of a text's meaning by another text' (Ivanic, 2004, p. 281) illustrates its complex nature.

The ambiguity of this notion also manifests itself in a diversity of types of plagiarism described in the research literature (e.g. Decoo, 2008; Harvard Guide to Using Sources, n.d; Lei \& Hu, 2015; Roig, 2001; Walker, 1998) which we summarized in Table 1 below.

Table 1. Types of plagiarism

\begin{tabular}{|c|c|c|}
\hline Type & Subtype & Definition \\
\hline \multirow[t]{2}{*}{$\begin{array}{l}\text { Word-for-Word } \\
\text { copying }\end{array}$} & Verbatim & $\begin{array}{l}\text { Word-for-word copying of large passages without in-line } \\
\text { acknowledgement of the source }\end{array}$ \\
\hline & Mosaic Plagiarism & Some parts rather than the whole text are copied word-by word \\
\hline \multirow[t]{4}{*}{ Paraphrase } & & Unacknowledged borrowing of ideas \\
\hline & Sham Paraphrasing & $\begin{array}{l}\text { Material copied verbatim from text and source acknowledged in-line } \\
\text { but represented as paraphrased. }\end{array}$ \\
\hline & Inadequate Paraphrasing & $\begin{array}{l}\text { The language of the new text, though paraphrased, is still very close } \\
\text { to and imitates the original }\end{array}$ \\
\hline & Illicit Paraphrasing & $\begin{array}{l}\text { Material paraphrased from text without in-line acknowledgement of } \\
\text { the source. }\end{array}$ \\
\hline $\begin{array}{l}\text { Illicit } \\
\text { Referencing }\end{array}$ & & One provides incorrect information about the source of a reference \\
\hline \multirow[t]{3}{*}{ Intentionality } & Intentional Plagiarism & One is aware of cheating \\
\hline & Cryptomnesia & Unconscious plagiarism \\
\hline & Other Plagiarism & $\begin{array}{l}\text { Material copied from another student's assignment with the } \\
\text { knowledge of the other student. }\end{array}$ \\
\hline \multirow[t]{3}{*}{ Peer plagiarism } & Purloining & $\begin{array}{l}\text { Assignment copied from another student's assignment without that } \\
\text { person's knowledge. }\end{array}$ \\
\hline & Ghost Writing & Assignment written by third party and represented as own work. \\
\hline & Recycling & Same assignment submitted more than once for different courses. \\
\hline $\begin{array}{l}\text { Internet } \\
\text { Plagiarism }\end{array}$ & & $\begin{array}{l}\text { Copying from an Internet source since the author can be anonymous } \\
\text { as opposed to offline sources. }\end{array}$ \\
\hline
\end{tabular}

With more than ten different types of plagiarism being identified so far the debate about the level of seriousness, degree of acceptance and awareness seems to be centred around the two types, i.e. copying word-for-word and paraphrasing. In this study, we follow Harvard Guide to Using Sources, which defines mosaic plagiarism as a kind of academic dishonesty when some parts of the text, namely more than ten consecutive words, are copied word-by word from the original source without due acknowledgement (Harvard Guide to Using Sources, n.d.). Following the opinion of 
Walker (1998), by illicit paraphrasing we understand academic dishonesty when the material is paraphrased from a text without in-line acknowledgement of the source.

Considering the recent technological advancements resulting in more materials becoming open-access which in turn might be an attraction for the students to plagiarise, research on plagiarism is of immediate relevance. Research on literature shows that there has been a variation through time in terms of how often students plagiarize. For example, the study of Karlins et al. (1988) gathered data on selfreported plagiarism revealing that only $3 \%$ of the students on a business course plagiarized an assignment. However, a study of Jackson in which the same method of data collection was employed, indicated an escalation in plagiarism, "with over 75 percent of students admitting to some cheating' (Jackson, 2006, p. 418). On the whole, data on plagiarism incidence are scarce due to its complex nature and different sources of information that form the basis of research studies investigating it - selfreporting by students or detection rates by instructors and computer tools. Karlins et al. (1988) more than two decades ago already emphasized the necessity to examine what students do rather than what they think / say they do concerning plagiarism as there might be significant differences between the two.

The lack of consensus on a comprehensive definition of plagiarism might add to more confusion in the understanding of this notion and conclusions concerning pedagogical implications. Students' ability to identify plagiarism in a text as well as their perceptions of this phenomenon need to be considered in the process of finding solutions to overcome this problem.

\subsection{Literature review}

Taking into account the complex nature of plagiarism and its longstanding history, since it might be as old as writing itself, the literature review showed that research on academic dishonesty or misconduct has taken different directions. We categorized the topics that addressed plagiarism into five groups.

The first group of studies looked into the perceptions of individuals on plagiarism. Among the investigated trends are studies aimed at identifying variations in viewpoints between undergraduate and graduate students (Brown, 1995), and students and teachers (Sivasubramaniam, 2004; Wilkinson, 2009; Yazici et al., 2011). In the latter case, it was concluded that teaching staff and students vary slightly in their attitudes towards cheating with teachers holding harsher views on the kind of punishment one may give for the cheating behavior (Wilkinson, 2009). Yazici et al. (2011) observed that the nature of the writing assignment also influences the perceptions of students as they find it less ethical to plagiarize on in-class writing tasks rather than home assignments.

A number of cultural studies have been conducted aimed at eliciting the differences in understanding of plagiarism in the Western individualistic versus collective Eastern cultures (e.g. Gu \& Brooks, 2008; Maxwell et al., 2008). The study of Maxwell et al. (2008) concluded that fewer Asian students as compared to Australian students 
viewed illicit paraphrasing and word-for-word copying as plagiarism. An argument that was put forward to explain such differences in understanding of plagiarism resided in the idea that in Asian countries learning mostly focuses on covering the contexts of textbooks while in Western - to a bigger extent on critical creative assignments (Hayes et al., 2005). The qualitative study of $\mathrm{Gu}$ and Brooks investigated the changes in perceptions of plagiarism in the period of fifteen months of ten Chinese international students studying in a British academic community (Gu \& Brooks, 2008). The researchers stated that plagiarism is a sociocultural phenomenon as their study showed that the students had to overcome emotional tensions which arose from the shift in their cognition, sense of identity and sociocultural values in relation to plagiarism.

The second group of studies focused on analyzing the reasons that trigger plagiarized behavior again based on the perceptions of individuals concerning plagiarism. Straw (2002) developed a taxonomy of such reasons. The first indicated reason among the top three was a lack of understanding on the side of the students which is qualified as unintentional plagiarism as they do not have in-depth knowledge of citation rules or do not easily differentiate between 'common knowledge' and 'previously reported information'. The second reported reason for plagiarism was efficiency gain when students find shortcuts in their attempt to strive for a better grade or to save time. Lastly, personal attitudes of students also contribute towards their involvement in plagiarism as some students might not see plagiarism as a serious misconduct or might even view it as clever and acceptable. In another study alienation of students from the teaching staff was also reported to trigger plagiarism as technology is increasingly replacing face-to-face communication and the number of students per teacher grows in the classroom, (Hayes, 2003). A study of SutherlandSmith which collected qualitative data on the perceptions of plagiarism from eleven teachers involved in teaching a first year English for Academic Purposes writing subject, voiced another opinion concerning the reasons for plagiarism. It was claimed that on a university level research is the key to promotion and not teaching. "Why bother [to track student plagiarists] when there's no future in it, particularly from the standpoint of promotion and self-interest' (Sutherland-Smith, 2005, p.93).

The third and largest group is represented by field correlative studies that looked into detection of plagiarism and the analysis of what type of students plagiarize most. There have been investigations based on gender difference (BavaHarji et al., 2016) with some evidence that male students, on average, plagiarize more than female. Other research studies attempted to identify the students of what majors tend to plagiarize most frequently. Meade (1992) revealed that among business, engineering, science and humanities faculties of 31 US universities the first two ranked top (87\% and $74 \%$ of the students respectively) as the students admitted they have plagiarized at least once throughout their university life. These findings were confirmed by BavaHarji et al. (2016) who also found out that plagiarism is more prevalent among students in technical rather than non-technical fields. However, students who are predominantly in the threat category and who might resort to plagiarism are ESL 
students as they are often involved in foreign language writing and they do not have native proficiency in it. In this light, research on English as a Medium of Instruction students and the students of other majors such as ELT might provide different results. Studies on age difference (BavaHarji et al., 2016; Straw, 2002) found that younger students cheat more often than older and mature students, which partly contradicts the conclusion made in the study of Brown (1995) which stated that undergraduate and graduate students 'show similar frequencies of unethical behavior'. Research on the correlation between academic ability and plagiarism has also brought conflicting results. Straw (2002) claimed that students with higher Grade Point Average (GPA) tend to plagiarize less than students with lower GPA while Roig (2001) has found no such pattern. Other studies established correlation between students' motivation and the prevalence of plagiarism practices. Gerdeman (2000) observed that those students who find the classroom subject matter unimportant or of no personal interest plagiarize more often than those who see the benefit or personal relevance of the writing task. Moreover, the students working with the teachers who regularly check the submitted assignments for plagiarism are reported to plagiarize less (Buckley et al., 1998) for the fear of punishment or embarrassment.

We classified the studies that analyzed possible ways to deter plagiarism into the forth group. Among pedagogical implications drawn in research was the idea that emerged in the USA in 1990s to establish university honor codes (Kibler, 1994). For example, Middle-East Technical University (METU) in Turkey adopted the honor code based on the following principles: 'The members of the METU community are reliable, responsible and honorable people who embrace only the success and recognition they deserve, and act with integrity in their use, evaluation and presentation of facts, data and documents' (METU Honor Code, n.d.). Some institutions like Duke University in the USA practice signing a pledge against academic misconduct by the students publicly in a ceremonial form. Since the university policy often serves as a benchmark of assessing plagiarized behavior, there have been case studies (e.g. Gullifer et al., 2014) investigating whether the students have read their universities' official documents on academic integrity and how well they understand them. The findings indicated that only half of the participants $(N=3405)$ had read the university policy on plagiarism and there was evident confusion regarding the practices that constitute plagiarism. Hansen et al. (2011) proposed educative rather than mere ethical or punitive measures that might help the teaching staff to discourage plagiarism among the students. Among the claimed measures, three of them can be highlighted. The first measure is giving creative assignments that require the students to use their critical thinking skills. The second measure is using a portfolio-based instruction that invites the students to submit not just the final version of the writing assignment but also all the written drafts as well as some copies of literature resources that guided the students in the writing process. The third measure is linked to the previous two and concerns continuous feedback-led assessment strategy where the assessment looks at the progress of the critical analysis. 
The last and fifth group is characterized by research efforts focused on attempting to determine students' criteria for plagiarism. In one such experiment, Hale (1987 as cited in Roig, 2001) provided the students with the original text and two paraphrased copies - one with and the other without correct citations. The students in the experiment were asked to compare each copy with the original and identify whether each of them is plagiarized or not. The study demonstrated that less than a quarter of the participants displayed difficulty in identifying plagiarism while most of them seemed to understand the difference between plagiarism and accepted writing practice. However, 'when grading papers, it is not uncommon to encounter instances in which students correctly attribute their written material to the original author, but their writing is too close to the original' (Roig, 2001, p. 308). Such empirically supported claims and also our personal observation of students' understanding of plagiarism during informal conversations with them and their behavior during writing assignments gave us ideas and inspiration for the current research study.

The above review showed that research aimed at determining variations in perceptions of plagiarism among students, correlation between what students think of plagiarism in theory and how they evaluate plagiarized pieces in practice as well as research focusing on different cultural and disciplinary contexts (as in the case of ELT field, e.g.) is exceedingly underrepresented at the moment. The current research study aims to address these issues in the Turkish context.

\section{Method}

\subsection{Aims and research questions}

The present study aimed to elicit ELT students' understanding of plagiarism, evaluate their ability to identify mosaic plagiarism and illicit paraphrasing in a written text as well as compare their perceptions on how serious and acceptable plagiarism is in theory and in practice.

The research questions addressed in the study are as following:

1. What are ELT students' perceptions of plagiarism?

2. What type of plagiarism - mosaic plagiarism or illicit paraphrasing - is seen as most serious by ELT students?

3. To what extent are ELT students able to identify plagiarism in a written text?

4. Is there any difference in how ELT students see plagiarism in theory and in practice?

\subsection{Participants}

As the main purpose of the present research was "to learn or understand the central phenomenon" (Creswell, 2012, p. 206) from the perspective of a specific group of students; purposeful sampling method, more specifically, homogenous sampling strategy was applied. The research participants were 32 second-year students of the 
Education department of Sakarya University, Turkey. Second-year students were chosen for this research study due to the fact that they were taking Research Methodology in ELT, a compulsory course offered in the fourth semester within an eight semester program. This course serves as a comprehensive introduction of preservice language teachers to the basic research concepts and problems encountered in ELT scientific investigation, individual step-by-step design and implementation of a small-scale research study. The medium of instruction and the language in which the research studies are written is strictly English during the course which is the students' second language. Since it has become conventional wisdom in academic research that second-language writers plagiarize more frequently than L1 writers (Pecorari, 2003, p. 318), it is of vital importance to investigate the challenges L2 writers have and their perceptions of plagiarism. The participants were similar to one another in terms of language proficiency and academic conduct as proved by their scores on the proficiency test given by the Department and by their Grade Point Averages (GPAs). All the students scored above 80 out of 100 on the proficiency test they took to pass the preparatory class. Eighty on this test is equal to B2 on Common European Framework of Reference for Languages (CEFR) as identified by the regulations of the Council of Higher Education in Turkey. Participants themselves indicated their own GPAs on the questionnaires and they were also verified from Students Affairs Department of the University. Participants' GPAs as indicators of their academic conduct range between 1.80 and 3.00 out of 4.00 . Students within this range are allowed to continue their studying and graduate. More precisely, students with a GPA that is lower than 1.80 are considered academically unsuccessful and those with a GPA that is higher than 3.00 are acknowledged as honor or high honor students, who similarly represent another extreme case. Thus, it can confidently be stated that participants were those "who can best help us understand our phenomenon", which characterizes homogenous sampling strategy (Creswell, 2012, p. 206). Prior to our research study, all the participants received one hour of instruction on ethics in research as part of their Research Methodology in ELT class. The syllabus of the course which was distributed to all the students together with the course-books also included an explanation of the instructors' policy on plagiarism. The syllabus provided a definition of plagiarism and informed the students that anti-plagiarism software would be used for all classroom assignments. Before the start of the data collection process ethical approval was sought internally from all the participants of our research before they were recruited. They were provided with our contact details, informed about the aims of this research study and were ensured about the confidentiality of the information they provide and the voluntary basis of their participation.

\subsection{Instruments and procedure}

The data were collected from a two-part questionnaire, semi-structured interviews and think-aloud protocols. In the first part of the questionnaire (see Appendix A) we formulated open-ended questions on students' general understanding of plagiarism. 
The students were also provided with three situations, two of which illustrated plagiarized behavior and one - non-plagiarized behavior (see Appendix B). The students were asked to state whether each situation represented plagiarism, rate how serious the plagiarism was and comment on whether they had done a similar thing in the past. In line with the study of Hale (1987), the second part of the questionnaire was practice-oriented and included four 150 -word texts. The first text was the original followed by three copies: a copy containing mosaic plagiarism from the original, a copy with illicit paraphrasing and a copy with no compromise of academic integrity. The texts featuring academic dishonesty were plagiarized according to the definition of plagiarism outlined in the literature review (Harvard Guide to Using Sources, n.d.; Walker, 1998). Mosaic plagiarism containing a sentence with 26 words copied consecutively word-for-word from the original text was placed in the middle of the first copy. The second copy was based entirely on illicit paraphrasing of the original text. All four texts were taken from the actual assignment for another course where one of the authors of the present research had detected these two types of plagiarism in their teaching practice. The students were asked to carefully read each text, compare the copies with the original and comment on the presence of plagiarism, its seriousness and acceptability. Since this study focused only on word-for-word vs. paraphrase plagiarism, only these two types were featured in our data collection tools.

After the questionnaires which were filled in anonymously by the students, 16 individual interviews were conducted with the research participants to collect more in-depth information. The participants were randomly selected for the interviews. Similar procedure was used in the interviews as in the questionnaire. In the first part of the interview the participants answered questions on their views on plagiarism. In the second part of the interview they were provided with four new texts, two of which contained either mosaic plagiarism or illicit paraphrasing, and were asked in the form of think-aloud protocols to comment on the presence of plagiarism, its seriousness and acceptability. The answers of each student in the first and second part of the interview were compared for discrepancies. The open-ended parts of the questionnaires and the transcribed interviews were coded for the reoccurring themes.

In this study qualitative rather than quantitative methods were employed as the aim of the study was to obtain detailed description of students' opinions and experiences concerning plagiarism and interpret the meaning of their actions when they are exposed to plagiarized texts.

\section{Results}

An analysis of the qualitative data gathered through the open-ended questionnaires, semi-structured interviews and think-aloud protocols according to the Data Analysis Spiral (Creswell, 2007) has revealed participants' perceptions of plagiarism, the level of their competence in identifying different types of plagiarism in written work, their opinions about seriousness and acceptability of two types of 
plagiarism under investigation, and the level of correspondence between their ways of seeing plagiarism in theory and in practice.

\subsection{Participants' perceptions of plagiarism}

Students' perceptions of plagiarism were analyzed in terms of their own definitions of the terminology, the reported motives behind students' acts of plagiarizing, and possible teacher strategies suggested by the participants to prevent students from gravitating towards plagiarism. Codes and categories that emerge from the data were qualitatively described, interpreted, and classified if necessary. These were either in vivo codes that were the participants' own words, or the labels the researchers composed to describe the information at its best (Creswell, 2007). Though the codes were counted preliminarily, the counts are not reported since it 'conveys a quantitative orientation of magnitude and frequency contrary to qualitative research' (Ibid., p. 152). However, counts and numbers have been explicitly stated to answer the relevant research questions when a quantitative perspective is eligible.

Students define plagiarism in different words, however all the definitions have revealed that they have an idea of what it means, which does not guarantee that they know the specific rules or apply the theory into practice. They collectively associate the term with the issue of morality and view plagiarism as immoral. The most frequently used word to define plagiarism is 'stealing'. Students underline presenting others' peculiar ideas and opinions as your own without giving any credit to the original text in their definitions of plagiarism. One student, for instance, defined the term as 'stealing opinions and labors of others and using them as our own' [Participant (P)15]. Another participant, in the interview, equated it with 'normal stealing', and added that 'it is a crime to be punished' [P12]. In a similar way, plagiarism was conceptualized as 'using ideas without taking permission of the owner' [P21]. Students' recurring emphasis on 'ideas' may suggest that they regard originality and effort put by authors to produce unprecedented mental work. Thus, taking permission, giving reference to the original source, and acknowledging the author are considered to be the responsibilities of the writers who are using a piece of information or idea belonging to someone else. In addition, some participants believe that plagiarizing is almost inevitable, and as one student claimed, in their opinion, 'unfortunately, anyone uses plagiarism in their research' [P17].

Regarding the reasons for students' plagiarizing, despite a common awareness of it being an ill act, they brought a number of points into the forefront. Table 2 demonstrates the codes that emerged from participants' speculations on possible motives behind students' resorting to plagiarism.

Table 2: Students' Reported Motives behind the Acts of Plagiarism

\begin{tabular}{ll}
\hline Theme & Codes \\
\hline Reasons for Plagiarizing & Lack of skills \\
& Time restriction \\
\hline
\end{tabular}


Inability to produce own ideas or expressions

Not taking referencing seriously

Desire to look cool

Getting higher grades

No intrinsic motivation

As can be seen in Table 2, participants held the idea that students mostly plagiarize due to some inadequacies or deficiencies. They reported that plagiarists may not be competent users of language or they may not be able to think up their own ideas. Additionally, having insufficient amount of time or a desire to complete an assignment quickly also leads students to plagiarize. Students' ambition to score higher on assignments is counted as another emerging code. To illustrate, one student wrote that 'sometimes I plagiarize from other texts because nothing comes to my mind and I need some ideas and I check other texts, sometimes I copy and paste because there are very well-developed sentences and they fit my text' [P23]. Some cases of plagiarism were attributed to affective factors including students' attitude, acting solely with instrumental motivation. Another student, to refer to students' general attitude towards plagiarism, stated that 'we think taking others' words and ideas without giving any reference is not a big deal' [P4]. As other participants associating the act of plagiarizing with affective factors, another student noted that they, as students, 'see a good paragraph about an important research and [they] want to use this paragraph as if [they] wrote, [and] to be 'cool', [they] don't use citations' [P7].

Besides the reasons for plagiarism, participants suggested certain teacher strategies to dissuade students from inclining to plagiarism. Table 2 outlines the participants' suggestions to deal with the phenomenon.

Table 2: Teacher Strategies against Plagiarism as Reported by the Participants

\begin{tabular}{ll}
\hline Theme & Codes \\
\hline Strategies against Plagiarism & Raising awareness \\
& Teaching citation rules \\
& Teaching on examples \\
Making plagiarists fail & Shortening assignments \\
Developing students' self-confidence & Prioritize original opinions \\
& Teaching how to use plagiarism checkers
\end{tabular}

The most frequently repeated strategy offered by the participants is raising awareness among students, since they believe, as said in one of the interviews, 'many (if not all) will act according to [the] rules [of plagiarism]' if they are well-aware. Some sample cases from the former assignments could be used, they suggested, to illustrate plagiarism, and the codes of citation and referencing could be taught through such representative pieces. They also proposed utilizing anti-plagiarism software to unravel plagiarized parts in a text. Following the identification of plagiarism, they opted for some sort of punishment involving failure in a course or university 
reprimand. As they attributed students' act of plagiarizing to the low level of competence in language, a need for developing self-confidence to foster success was also emphasized. Furthermore, they argued that some adjustments made to the structure, nature, or length of assignments might also work. To exemplify, one student indicated that 'I think, firstly teachers should inform their students that 'your opinions are much more important for me, do not try to take someone else's opinion' like that' [P10]. In the follow-up interviews, the same suggestion was repeated. One interviewee, for instance, asserted that 'comments should be demanded in the assignments rather than the information' [P7]. So, participants believe that assignments that are thought-provoking are of higher benefit than those asking for a review of the literature. Apart from all these, some participants viewed the problem of plagiarizing out of teachers' control as represented in the statement of another participant; 'if a student has decided [to plagiarize], sometimes there is nothing to do for teachers' [P9].

\subsection{Students' opinions on seriousness and acceptability of two types of plagiarism}

Participants were inquired about their opinions on two different types of plagiarism, namely mosaic plagiarism and illicit paraphrasing. They were asked to assess these two types in terms of seriousness and their assessment of acceptability. All the participants, except for one, marked mosaic plagiarism as serious at varying degrees. 15 out of 32 participants rated it 'very serious,' nine of them 'serious', and three 'quite serious'. Four participants did not comment on the seriousness of wordfor-word plagiarism. One student, to illustrate, considered it as 'the most serious level of plagiarism' [P13]. Another student likened mosaic plagiarism to 'taking someone's money or anything without asking them' to stress its high level of seriousness [P20]. A distinction was also made between the two types by defining the mosaic plagiarism as 'not only copy[ing] the idea but also the words' [P32]. Despite their strict assessment of mosaic plagiarism, half of the participants reported that they resorted to it at least once in the past. Twelve students claimed that they have not plagiarized word-forword so far while four students did not comment on their past experience. Most of the students explained their past experience of plagiarizing through lack of knowledge or awareness. The only student who viewed mosaic plagiarism as 'not serious' wrote that 'I do not think it is a serious plagiarism. I have done similar things in my previous classes, However, I took not more than a few words, they were not complete sentences' [P4].

It has been yielded that illicit paraphrasing is seen less serious by the participants. Seven out of 32 participants think that illicit paraphrasing is 'not serious' and it is acceptable. Two participants stated that they could not determine its seriousness while five of them avoided commenting on it. On the other hand, all the other remaining participants rated it as serious at different levels. One participant assessed it as 'quite serious', 14 as 'serious', and three as 'very serious'. One participant, who views illicit paraphrasing as 'very serious', noted that 'paraphrasing the text does not give you the right not to mention the author, because the idea still does not belong to 
you even if the words do' [P32]. However, some others advocated a contrary opinion and argued that 'paraphrasing shouldn't be seen as plagiarism because you just try to get the main idea, but you write it in your own words' [P11]. Similar to the mosaic plagiarism, 16 out of 32 participants have had an experience of the latter type of plagiarism. Some of them identified their act as acceptable on the grounds that the idea was good enough for [their] needs' [P6] or what s/he paraphrases is 'a common opinion' [P30]. Six participants did not comment on their past experience of illicit paraphrasing and the remainder claimed that they have not done it before. It should also be underlined that students made a difference between high school and university years, and explained their earlier acts through their inefficiency.

Participants' perception of acceptability of plagiarism is also associated with some factors other than its type. The most prominent determiner has been claimed to be the notion of intentionality. In other words, participants tend to tolerate plagiarism if it is owing to students' ignorance, lack of knowledge of proper referencing or awareness; however, intended plagiarism that is done knowingly and willfully is not accepted. In an interview, one of the participants said that 'some students are not aware but if he does it intentionally, I will not accept it but I don't know how to decide, similarity report might be?' [P26]. Though intentionality plays a major role in their assessment of acceptability, they could not come up with a justifiable way of testing intentionality. Participants' subjective opinions on the educational stage of students, the extent to which they are creative, whether it is at paraphrasal level or not, and whether the problem is only with citation rules or not are the other factors that shape the participants' decisions concerning the acceptability of plagiarism.

\subsection{Identification of plagiarism and the match between theory and practice}

It was revealed that only eight students out of 32 participants know the rules of proper referencing because they considered the paraphrased extract with the name of the author but without the page number as plagiarism, which is normally not considered as misconduct. Additionally, participants were mostly not successful in identifying plagiarism. Only two of the participants were able to accurately identify and explain both types of plagiarism. Eleven participants failed to identify verbatim plagiarism while three - for plagiarism at paraphrase level. Seven participants were not able to identify any types of plagiarism. On the other side, though some participants could identify plagiarism, they were not able to provide correct explanations. For instance, one participant wrote that 'there is no reference in the text. There are some statements which are very certain, and according to what these statements are given?' [P4] So, it is obvious that the student examined the text in terms of the rules of proper referencing and citing, in other words, s/he did not compare it with the original one for the similarities between the two texts. Although that participant stated that the text is plagiarized, s/he could not properly explain why and in what ways it is so. Five students could identify plagiarism in both texts; however, they could not explain any of them accurately. Five students identified mosaic plagiarism but could not describe how the text is plagiarized, and the same 
number of students identified illicit paraphrase but failed to explain why it is so. In the interviews, similarly, four participants could not identify any types of plagiarism and two of them failed in recognizing mosaic plagiarism. To recapitulate, other than two, all the participants had problems with either identifying or explaining plagiarism in written work somehow although they were able to define what plagiarism is in general.

Students' previous considerations on plagiarism in the first part of the questionnaires and in the semi-structured interviews were compared with their real practices in the second part of the questionnaire and in the think-aloud protocols. The results showed that for almost all the participants who could at least identify one type of plagiarism -though they may not be able to explain it- theory matches with practice. To illustrate, when asked if he would accept any types of plagiarism as a prospective teacher, one student stated in the interview that 'to some extent I may accept, to get an idea, plagiarism is acceptable'. While assessing the acceptability of the text that is plagiarized word-for-word, the student detected the sentence that was directly taken from the original text without any references, and said that it is plagiarized but not serious, I would accept it, my score is 1 [not serious]' [P5]. Thus, this specific student's opinions about the acceptability of plagiarism in theory perfectly match his real practice similar to many others.

\section{Discussion}

The main sources of inspiration for the present study were the ambiguity prevailing in the conception of plagiarism and the assumptions that students in the local context do not seem to be competent in identifying plagiarism. Furthermore, as yielded by the study conducted by Meade (1992), some differences might occur between students majoring in different fields. Another aim was to respond to the need of revealing correspondence between students' opinions and real practices since some discrepancies might be observed between students' perceptions and practices as suggested by Karlins et al. (1988). The results have proved the original hypotheses and the necessity of carrying out such a study to scrutinize the controversial, deeplyrooted phenomenon of plagiarism.

In the literature, it has already been well-established that plagiarism has remained complex and quite diverse views exist around the term (Decoo, 2008; Ivanic, 2004; Lei $\& \mathrm{Hu}, 2015)$. Likewise, in relation to the first two research questions of our study the results manifested that students hold diversified views surrounding the definition, acceptability, and seriousness of different types of plagiarism. Although almost all the students have lucid attitudes towards the unethicality of plagiarism, they differ from one another with respect to their evaluation of acceptability and seriousness of plagiarism. The study revealed that mosaic or word-for-word plagiarism is seen as more serious than illicit paraphrasing. There was only one student in the sample who does not consider mosaic plagiarism as a serious misconduct and would accept it as a prospective teacher. Moreover, some students accept illicit paraphrase as a source of 
inspiration or an innocent way of getting some ideas about a topic. It was also quite notable that despite clearly stated negative attitudes they have, almost half of the students reported that they have plagiarized in their studies at least once so far. This supported the findings of Jackson (2006) who found out that $75 \%$ of the students have somehow gravitated to plagiarism. Thus, as Karlins et al. (1988) asserted, significant differences between students' perceptions and real practices might occur. This raises the question of why students are inclined to plagiarize and revitalizes the need for a thorough investigation of the motives behind their acts.

The exploration of the reasons for students' cases of plagiarism has brought up a prominent pedagogical implication. Students believe that assignments that require them to be creative and generate their own unique opinions may refrain them from plagiarizing. Therefore, as Hansen et al. (2011) implicated, teachers ought to assign thought-provoking tasks and encourage students to think creatively. It was also found in the present study that students resort to plagiarism due to lack of a clear understanding of policies of academic honesty. In a similar vein, Straw (2002), marked this issue as one of the top reasons for plagiarism in the taxonomy. Hence, the need for prioritizing academic honesty, raising awareness among students, and teaching the codes of academic ethics has been once again underlined. In order to extinguish the ambiguity in the meaning and rules of plagiarism, specifying the norms and informing students about them may contribute to a decrease in the number of plagiarism cases. Due to the elusive nature of the phenomenon, it might also be substantiated that teaching the notion on examples rather than merely presenting the theoretical knowledge as was carried out in Research Methodology in ELT course the students were taking at the time the data for this study were collected may be more beneficial. The concrete result that more than two-thirds of the participants do not know the rules of referencing in spite of the abovementioned course they were taking also solidifies the validity of this implication. Yet another noteworthy result pointing to the role of intrinsic motivation implies that teachers should maintain students' engagement in assignments. Practitioners are expected to clarify the benefits of and motives behind the tasks they assign. The implication supports the study of Gerdeman (2000) calling attention to the correlation between the prevalence of plagiarism and students' level of motivation. Lack of proficiency in the target language was another reason for plagiarism that was reported by the participants of the present study too. It foregrounds the possible impact of students' majors on their behaviors of plagiarism (Meade, 1992). Since the medium of instruction in ELT departments and in general English courses is English, the finding may allude to the recommendation that teachers adjust the requirements of a task in compliance with students' language proficiency level.

In relation to the third research question, although a number of studies including Hale (1987) has demonstrated that only a minority group of students have difficulties with identifying plagiarism, our personal observations as well as the results of the present study showed that the students are not competent in identifying and explaining plagiarism. However, all the students were observed to have some ideas 
about what plagiarism is and it may be attributed to the instruction they had received in Research Methodology in ELT course. The discrepancy between the two suggests that their ideas do not depend on a solid base of knowledge, thus it again crystalizes a need for further training in plagiarism and academic ethics. In relation to the fourth research question, the significant match between theory and practice also unravels that properly shaping students' perceptions and providing them with training could considerably contribute to a decrease in the number of the cases of plagiarism.

In terms of limitations of the present study, some caveats should also be noted for a better appreciation of the results and implications drawn on them. First of all, in mosaic plagiarism where the statement that is extracted from the original piece is inserted may affect the results as it may affect its perceptual saliency. In the present study, it was located in the middle of the text, so the low level of students' recognition might be attributed to the issue of attention rather than their skills in identifying plagiarism. Secondly, interviewers' intervention during the think-aloud protocols may also guide the students and play a role in their interpretation of plagiarism. Furthermore, the length and content of the passages may have an effect on the results as well. In the present study, all the texts were from students' own field, thus it may foster their comprehension and give them a chance to focus more on the language rather than allocating some time to process the input. Lastly, since this study is a qualitative research, the results might not necessarily be generalized to other contexts. Thus, the participants' familiarity with academic writing, type of instruction and syllabus within Research Methodology in ELT courses might influence students' perceptions of plagiarism.

\section{Conclusions}

ELT undergraduate students' perceptions of plagiarism, their competence in identifying and explaining it, and possible matches or mismatches between theory and practice has been investigated in the present study. The results suggest that students have all negative attitudes towards plagiarism although their views may differ with regards to the issues of seriousness and acceptability of plagiarism. They were not efficient in identifying and explaining the concrete cases of plagiarism, thus it suggests that further training and awareness-raising activities are required. Their perceptions shape their real practices to a significant extent; hence, it seems crucial to build a clear understanding of the notion among students to control their future behaviors. The study sheds light on the phenomenon of plagiarism in ELT departments in the Turkish context and provides significant implications both for practitioners and students. However, the subject of plagiarism is still an area in which further research is necessitated.

The study aimed at disclosing students' perceptions and their competencies in identification and explanation of plagiarism; however, students' actual behaviors of plagiarism in their submitted assignments might also be explored in the forthcoming studies. Another interesting research direction might be a comparative exploration of teachers' and students' perceptions in the local context of Turkey. A study that 
compares the views of students majoring in different departments in Turkey or at different degrees e.g. BA, MA, MS, or PhD might also yield unprecedented results. Lastly, a study on plagiarism could be conducted at a larger scale within the quantitative research paradigm to reach more generalizable findings.

\section{References}

BavaHarji, M., Chetty, T. N., Ismail, Z. B., Letchumanan, K. (2016). A Comparison of the Act and Frequency of Plagiarism between Technical and Non-Technical Programme Undergraduates. English Language Teaching, 9(4), 106-118.

Brown, B. S. (1995). The academic ethics of graduate business students: a survey, Journal of Education for Business, 70(3), 151-156.

Buckley, M. R., Wiese, D. S., Harvey, M. G. (1998). An investigation into the dimensions of unethical behavior. Journal of Education for Business, 73(5), 284-290.

Creswell, J. W. (2007). Qualitative Inquiry and Research Design: Choosing Among Five Traditions (2nd edition). Thousand Oaks, CA: Sage.

Creswell, J.W. (2012). Educational Research: Planning, Conducting and Evaluating Quantitative and Qualitative Research (4th edition). Boston: Pearson.

Decoo, W. (2008). Substantial, verbatim, unattributed, misleading: Applying criteria to assess textual plagiarism. In Student plagiarism in an online world: Problems and solutions. IGI Global. 228-243

Fishman, T. (2009). We know it when we see it is not good enough: Toward a standard definition of plagiarism that transcends theft, fraud, and copyright. Proceedings of 4th Asia Pacific Conference on Educational Integrity (4APCEI) (pp. 1-5). Wollongong, Australia: University of Wollongong NSW Australia.

Gerdeman, R. D. (2000). Academic Dishonesty and the Community College. ERIC Digest.

Gu, Q., Brooks, J. (2008). Beyond the accusation of plagiarism. System, 36(3), 337-352.

Gullifer, J., Tyson, G. A. (2010). Exploring university students' perceptions of plagiarism: A focus group study. Studies in Higher Education, 35(4), 463-481.

Hansen, B. (2003). Combating plagiarism. The CQ Researcher, 13(32), 775-792.

Hayes, N. (2003). Alienation and plagiarism: Coping with otherness in our assessment practice. Working Paper. The Department of Organisation, Work and Technology, Lancaster University. Retrieved from http://eprints.lancs.ac.uk/48693/

Hayes, N., Introna, L. D. (2005). Cultural values, plagiarism, and fairness: When plagiarism gets in the way of learning. Ethics \& Behavior, 15(3), 213-231.

Harvard Guide to Using Sources (n.d). Retrieved from https://usingsources.fas.harvard.edu/

Ivanic, R. (2004). Intertextual practices in the construction of multimodal texts in inquirybased learning. Uses of intertextuality in classroom and educational research, 279-314.

Jackson, P. A. (2006). Plagiarism instruction online: Assessing undergraduate students' ability to avoid plagiarism. College \& Research Libraries, 67(5), 418-428.

Karlins, M., Michaels, C., Podlogar, S. (1988). An empirical investigation of actual cheating in a large sample of undergraduates. Research in Higher Education, 29(4), 359-364.

Kibler, W. L. (1994). Addressing academic dishonesty: What are institutions of higher education doing and not doing? NASPA Journal, 31(2), 92-101.

Lei, J., \& Hu, G. (2015). Chinese university EFL teachers' perceptions of plagiarism. Higher Education, 70(3), 551-565. 
Maxwell, A., Curtis, G. J., \& Vardanega, L. (2008). Does culture influence understanding and perceived seriousness of plagiarism?. International Journal for Educational Integrity, 4(2).

Meade, J. (1992) Cheating: is academic dishonesty par for the course? Prism, 1(7), 30-32.

METU Honour Code (n.d.). Retrieved from http://fbe.metu.edu.tr/metu-honour-code/

Pecorari, D. (2003). Good and original: Plagiarism and patchwriting in academic secondlanguage writing. Journal of second language writing, 12(4), 317-345.

Roig, M. (2001). Plagiarism and paraphrasing criteria of college and university professors. Ethics \& Behavior, 11(3), 307-323.

Sivasubramaniam, S. D. (2004). Plagiarism amongst bio-medical science undergraduates concerned academics versus ignorant students. Proceeding of 1st International Conference on Plagiarism. JISC, 205-224.

Straw, D. (2002) The plagiarism of generation 'why not?', Community College Week, 8 July, 14(24), 4-7.

Sutherland-Smith, W. (2005). Pandora's box: Academic perceptions of student plagiarism in writing. Journal of English for Academic Purposes, 4(1), 83-95.

Walker, J. (1998). Student plagiarism in universities: What are we doing about it? Higher Education Research \& Development, 17(1), 89-106.

Wilkinson, J. (2009). Staff and student perceptions of plagiarism and cheating. International Journal of Teaching and Learning in Higher Education, 20(2), 98-105.

Yazici, A., Yazici, S., \& Erdem, M. S. (2011). Faculty and student perceptions on college cheating: Evidence from Turkey. Educational Studies, 37(2), 221-231.

\section{Appendix A. Open-ended questionnaire on students' perceptions of plagiarism}

\section{Dear friend,}

we are conducting a research study aimed at eliciting students' understanding of plagiarism. All information provided by you is completely confidential - so, please, be honest in your answers. In case you have further questions concerning this study, you can contact the researchers by writing to one of the following e-mails: $x @ x, x @ x$

I accept to participate in this research study on a volunteer basis.

Signature:

Your year of study:

Please, provide answers in the allocated spaces.

1. How would you define plagiarism?

2. Why, in your opinion, do students plagiarize?

3. Imagine a situation in which you copy a part of the text word-by-word from a book or another student's paper without indicating the authorship and putting quotation marks. Is it plagiarism? How serious is it? Have you ever done a similar thing in the past and why / why not? Please, answer each question.

4. Imagine a situation in which you paraphrase a part of the text from a book or another student's paper without indicating the authorship. Is it plagiarism? How serious is it? Have you ever done a similar thing in the past and why / why not? Please, answer each question. 
5. Imagine a situation in which you paraphrase a part of the text from a book or another student's paper. You indicated the name of the author but not the page number. Is it plagiarism? How serious is it? Have you ever done a similar thing in the past and why / why not? Please, answer each question.

6. Do you consider it as plagiarism if you borrow ideas or text from a website where the author is not indicated rather than from a book? Why / Why not?

7. What can be done by the teacher to eliminate the desire to plagiarize among the students?

8. Will you try to detect plagiarism among your students' papers? Why? / Why not? State reasons. Will you accept any forms of plagiarism?

\section{Appendix B. Texts for plagiarism detection}

Please, read each text carefully, compare it with the original and comment out loud on the given questions.

\section{Original}

The strong relation between autonomy and motivation is based on the presumption that more autonomous a learner is, the more motivated they are (Dickinson 1995). Motivation is defined as 'effort plus desire to achieve, plus favourable attitudes towards learning the language' (Gardner 1983; 1991; 2004). Thus it is supposed that autonomy is a basic means to increase motivation. It may be concluded that in the ideal teaching/learning environment they present two sides of one coin where autonomy is the vehicle for increasing motivation and increased motivation in turn contributes to the further development of autonomy. One of the basic goals of the professional educators is to raise learners' motivation in the teaching/learning process which will guarantee higher achievements. Learner autonomy, if done correctly, is expected to help students develop a positive perception of their selves.

\section{Text 1}

Learner autonomy is widely recognized as a key element of language learning and has received a great deal of attention from L2 researchers and practitioners over the years (e.g., Dam, 2011). Autonomy takes a variety of forms that can be promoted in different ways and to different degrees depending on each learner and learning context. Autonomy has been defined as "the ability to take charge of one's own learning" (Holec, 1981, p. 3). While learner autonomy emphasizes independence and selfregulation, it is not the same as self-study or self-access learning. On the other hand, autonomy is the vehicle for increasing motivation and increased motivation in turn contributes to the further development of autonomy. To foster autonomy, teachers need to use affective strategies, such as reducing learner anxiety and encouraging learners to monitor their own stress and emotions. 


\section{Text 2}

Holec's (1981) definition of learner autonomy has proved remarkably robust and remains the most widely cited definition in the field. Variations on this definition abound. 'Ability' is often replaced by 'capacity' (a term used by Holec elsewhere), while 'take charge of ' is often replaced by 'take responsibility for' or 'take control of ' one's own learning (terms also used by Holec). The key element in definitions of this kind is the idea that autonomy is an attribute of learners, rather than learning situations. The strengthening of this view, based on the assumption that learners do not develop the ability to self-direct their learning simply by being placed in situations where they have no other option, is one of the more significant developments in the definition of learner autonomy over the past 30 years.

\section{Text 3}

Learner autonomy, especially in the field of foreign language learning was clearly articulated in the 1979 report prepared by Holec for the Council of Europe under the title of Autonomy in Foreign Language Learning. As for the philosophical background of learner autonomy, the starting point to encourage learners to become more autonomous is to have them accept the responsibility for their own learning. There is a powerful positive correlation between autonomy and motivation. The latter is commonly defined as a combination of desires and positive attitudes towards achieving a learning goal. However, there is still debate in academia concerning the nature of this correlation which reminds of a philosophical chicken-and-egg matter. Does motivation drive autonomy or is it a strong ability to be autonomous that contributes to stronger learning motivation?

\section{Copyrights}

Copyright for this article is retained by the author(s), with first publication rights granted to the Journal.

This is an open-access article distributed under the terms and conditions of the Creative Commons Attribution license (CC BY-NC-ND) (http://creativecommons.org/licenses/by-nc-nd/4.0/). 